\title{
High-energy resummed distributions for the inclusive Higgs-plus-jet production at the LHC
}

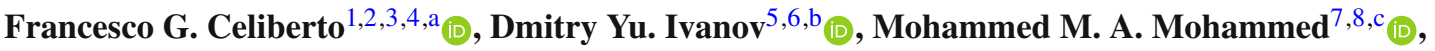 \\ Alessandro Papa ${ }^{7,8, \mathrm{~d}_{1}}$ \\ ${ }^{1}$ Dipartimento di Fisica, Università degli Studi di Pavia, 27100 Pavia, Italy \\ 2 Istituto Nazionale di Fisica Nucleare, Sezione di Pavia, 27100 Pavia, Italy \\ ${ }^{3}$ European Centre for Theoretical Studies in Nuclear Physics and Related Areas (ECT*), 38123 Villazzano, Trento, Italy \\ ${ }^{4}$ Fondazione Bruno Kessler (FBK), 38123 Povo, Trento, Italy \\ ${ }^{5}$ Sobolev Institute of Mathematics, 630090 Novosibirsk, Russia \\ ${ }^{6}$ Novosibirsk State University, 630090 Novosibirsk, Russia \\ ${ }^{7}$ Dipartimento di Fisica, Università della Calabria, 87036 Arcavacata di Rende, Cosenza, Italy \\ ${ }^{8}$ Istituto Nazionale di Fisica Nucleare, Gruppo collegato di Cosenza, 87036 Arcavacata di Rende, Cosenza, Italy
}

Received: 5 August 2020 / Accepted: 15 March 2021 / Published online: 7 April 2021

(C) The Author(s) 2021

\begin{abstract}
The inclusive hadroproduction of a Higgs boson and of a jet, featuring large transverse momenta and well separated in rapidity, is proposed as a novel probe channel for the manifestation of the Balitsky-Fadin-Kuraev-Lipatov (BFKL) dynamics. Using the standard BFKL approach, with partial inclusion of next-to-leading order effects, predictions are presented for azimuthal Higgs-jet correlations and other observables, to be possibly compared with experimental analyses at the LHC and with theoretical predictions obtained in different schemes.
\end{abstract}

\section{Introduction}

The Balitsky-Fadin-Kuraev-Lipatov (BFKL) [1-4] approach is a consistent framework for the theoretical study in perturbative QCD of semi-hard processes [5], where the scale hierarchy, $s \gg Q^{2} \gg \Lambda_{\mathrm{QCD}}^{2}$ holds, $s$ being the squared center-of-mass energy, $Q$ the hard scale given by the process kinematics and $\Lambda_{\mathrm{QCD}}$ the QCD mass scale. For these processes, large energy logarithms enter the perturbative series with a power increasing with the perturbative order and compensate thereby the smallness of the strong coupling, $\alpha_{s}$, thus calling for an all-order resummation. Within the BFKL approach, such a resummation is now amenable both in leading (LLA) and next-to-leading (NLA) approximations, and

\footnotetext{
a e-mail: francescogiovanni.celiberto@unipv.it (corresponding author)

be-mail: d-ivanov@math.nsc.ru

c e-mail: mohammed.maher@unical.it

d e-mail: alessandro.papa@ fis.unical.it
}

some steps towards the extension of the formalism beyond the NLA have also been done (see, e.g. Ref. [6]).

In the BFKL framework, the cross section of hadronic processes takes a peculiar factorized form, combining two impact factors, related to the transition from each colliding particle to the final-state object produced in the respective fragmentation region, and a process-independent Green's function. The latter is determined by an integral equation, whose kernel is known at the next-to-leading order (NLO) both for forward scattering (i.e. for $t=0$ and color singlet in the in the $t$-channel) $[7,8]$ and for any fixed, not growing with $s$, momentum transfer $t$ and any possible two-gluon color state in the $t$-channel [9-13].

Unfortunately, the list of impact factors known in the NLO is very short: 1) colliding-parton (quarks and gluons) impact factors [14-17], which represent the common basis for the calculation of the 2) forward-jet impact factor [18-22] and of the 3) forward light-charged hadron one [23], 4) the impact factor describing the $\gamma^{*}$ to light-vector-meson leading twist transition [24], and 5) the $\gamma^{*}$ to $\gamma^{*}$ transition [25-32]. This limits considerably the number of reactions which can be studied fully in the NLA BFKL approach. To enlarge this number, one has to resort to partial inclusion of NLA effects, by taking the two impact factors, or just one of them, in the leading-order (LO), using though the NLA BFKL Green's function.

Putting together full and partial NLA analyses, a respectable number of semi-hard reactions have been studied so far (see Refs. [33,34] for a review): the diffractive leptoproduction of two light vector mesons [35-38], the total cross section of two highly-virtual photons [39], the inclusive hadropro- 
duction of two jets featuring large transverse momenta and well separated in rapidity (Mueller-Navelet channel [40]), for which several phenomenological studies have appeared so far [41-57], the inclusive detection of two light-charged hadrons [58-60], three- and four-jet hadroproduction [6169], $J / \Psi$-jet [70], hadron-jet [71-74], Drell-Yan-jet [75,76] and heavy-quark pair photo- $[77,78]$ and hadroproduction [79].

Another engaging direction is represented by the possibility of probing the proton structure at low- $x$ through the BFKL resummation. More in particular, the emission of a single forward particle in lepton-proton or proton-proton scatterings offers us the chance to define an unintegrated gluon distribution (UGD) in the proton, written as a suitable convolution of the BFKL gluon Green's function and of a non-perturbative proton impact factor. Formerly used for the investigation of DIS structure functions [80], the UGD has later been studied via the exclusive diffractive electroproduction of a single light vector meson [81-86] at HERA and via the forward inclusive Drell-Yan production [87-89] at LHCb. Then, determinations of collinear parton distribution functions (PDFs) at NLO and next-to-NLO (NNLO) fixed-order calculations, improved via the inclusion of NLA small- $x$ effects, were proposed in the last years [90-92]. Quite recently, a model calculation of unpolarized and polarized transverse-momentum-dependent (TMD) gluon distributions effectively encoding a BFKL-driven input on small- $x$ tails was performed [93].

In this work we introduce and study with NLA BFKL accuracy a novel semi-hard reaction, i.e. the concurrent inclusive production of a Higgs boson and a jet:

$$
\begin{aligned}
& \operatorname{proton}\left(p_{1}\right)+\operatorname{proton}\left(p_{2}\right) \rightarrow H\left(\vec{p}_{H}, y_{H}\right)+\mathrm{X} \\
& \quad+\operatorname{jet}\left(\vec{p}_{J}, y_{J}\right),
\end{aligned}
$$

emitted with large transverse momenta, $\vec{p}_{H, J} \gg \Lambda_{\mathrm{QCD}}$, and separated by a large rapidity gap, $\Delta Y=y_{H}-y_{J}$. In Fig. 1 we present a pictorial view of this process, in the case when the tagged object in the forward (backward) rapidity region is the Higgs boson (jet).

For a Higgs boson with mass $M_{H}=125.18 \mathrm{GeV}$, the longitudinal-momentum fraction of the parent proton carried by the struck gluon is rather small, down to $x \sim 10^{-4} \div 10^{-3}$, making it possible to give a description at the hand of the BFKL resummation. Recently, a systematic framework to implement both the BFKL and the Sudakov resummations for the Higgs boson plus jet production [94], based on the TMD factorization, has been developed. The dominant partonic subprocess for the inclusive Higgs production at the LHC is represented by the gluon-gluon fusion, $g g \rightarrow H$, where the Higgs couples to gluons via a (top) quark loop, with coupling proportional to the (top) quark mass $M_{t}$. In our proposal, following Ref. [95], we adopt a kinematics which strictly

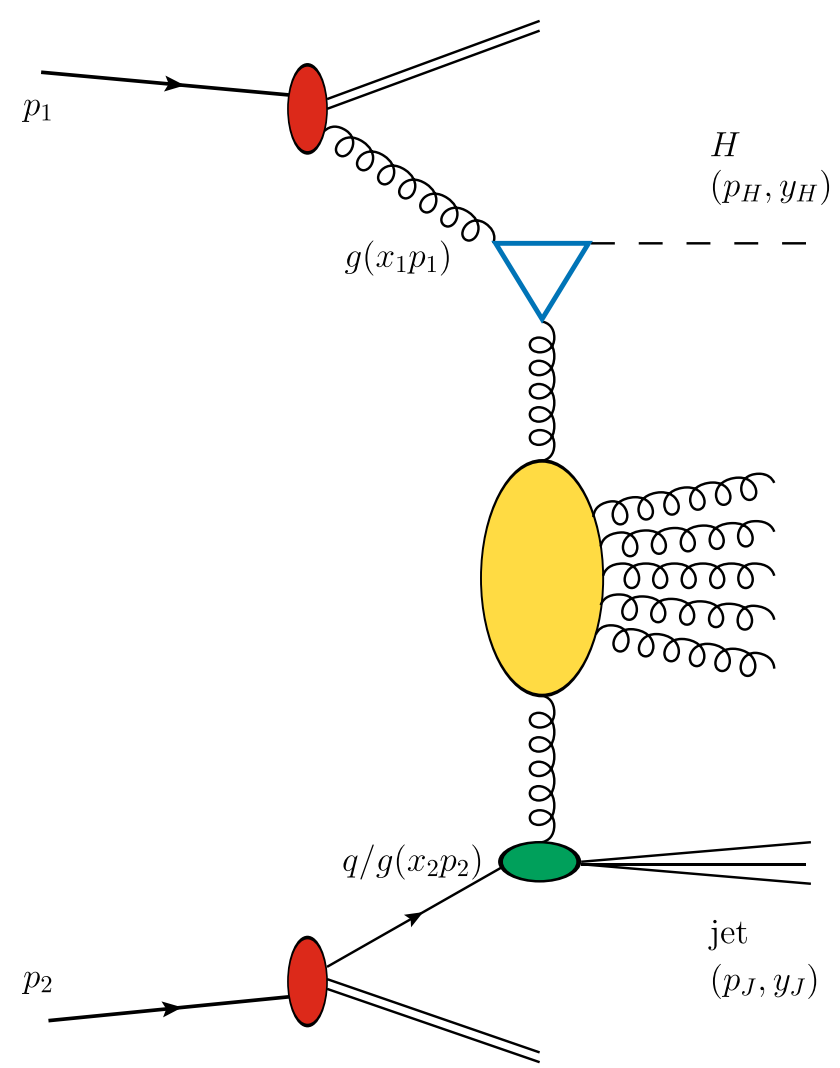

Fig. 1 Schematic representation of the inclusive Higgs-jet hadroproduction

respects the semi-hard regime, with the hard-scale set by the Higgs and top-quark masses, and the Higgs and jet transverse momenta satisfying the condition, $p_{H, J}^{2} \simeq M_{H}^{2}$. Moreover, to avoid the appearance of Sudakov double logarithms (see, e.g., Ref. [96]), unraveling only the high-energy ones, we introduce suitable cuts on transverse momenta to prevent the back-to-back emission of the Higgs and the jet, or to make this kinematical region marginal with respect to the remaining phase space. The tag of a jet in the peripheral regions of the detectors insures the existence of a large rapidity interval, $\Delta Y=y_{H}-y_{J} \simeq \ln \left(s / Q^{2}\right)$, with $Q^{2}$ a typical hard-scale value. In our analysis we do not include the resummation of Higgs transverse-momentum logarithms, which has been considered in several recent studies [97-101].

The key ingredient, needed for the study of our process in the BFKL approach, is the impact factor portraying the transition from a parton to a final-state Higgs boson, in the scattering off a Reggeized gluon. At the LO, the initial-state parton can only be a gluon. ${ }^{1}$

We will give predictions for cross section and correlations between the azimuthal angles of the Higgs and the jet in a

\footnotetext{
${ }^{1}$ Recently, the Higgs impact factor has been calculated also to the NLO [102].
} 
theoretical setup where NLA BFKL effects are included at the level of the Green's function.

The motivation for this work is twofold: on the phenomenological side, we want to calculate the cross section and to study the angular distributions of the process (Fig. 1) at LHC energies. Note that for that case the final-state objects to be identified are within current experimental reach of the LHC; in particular, the detection of the Higgs can profit by the well tried tools developed for its discovery. On the theoretical side, our approach is in a sense complementary to the most common ones devoted to Higgs production, where high-energy (or small- $x$ ) effects are possibly included as an improvement with respect to fixed-order calculations in collinear-factorization (see, e.g., Refs. [103,104] where the Altarelli-Ball-Forte (ABF) small $x$ resummation formalism is adopted [105-111], and Ref. [112]). Here, the view is reversed: we consider just high-energy effects, in the kinematical range where they only matter. Our results, which are the first for this kind of process encoding NLA BFKL effects, can therefore be used as a term of comparison for the other approaches, and contribute thereby to an improvement of our understanding of strong interactions. Moreover, the notorious problem of the NLA BFKL corrections, i.e. that they are large and opposite in sign with respect to the LLA, should not affect severely the determination of azimuthal correlations, ${ }^{2}$ due to the large energy scale provided by the Higgs mass.

The main theoretical limitation of the present work is that the impact factor for the Higgs production is taken at the LO, although, as explained later, some NLO terms predictable on the basis of renormalization group analysis, have been included in our calculation. This may seem reductive, especially in consideration that Higgs-plus-jet production was already calculated in QCD at the NLO $[115,116]$ and even in NNLO QCD [117-120] through the Higgs effective field theory (HEFT) [121]. We believe that this limitation does not spoil the global picture, since in the high-energy limit the NLA effects in the BFKL Green's function dominate over those in the impact factors. Nonetheless, the inclusion of NLO corrections to the Higgs impact factor is doable, though not trivial, and could be considered in future publications.

The paper is organized as follows: Sect. 2 is to set the theoretical framework up; Sect. 3 is devoted to our results for cross sections and azimuthal-angle correlations as a function of the rapidity interval, $\Delta Y$, between the tagged final objects (the Higgs boson and the jet); Sect. 4 carries our closing statements and some outlook.

\footnotetext{
${ }^{2}$ For ratios of azimuthal correlations it was shown that NLO effects are generally milder $[113,114]$.
}

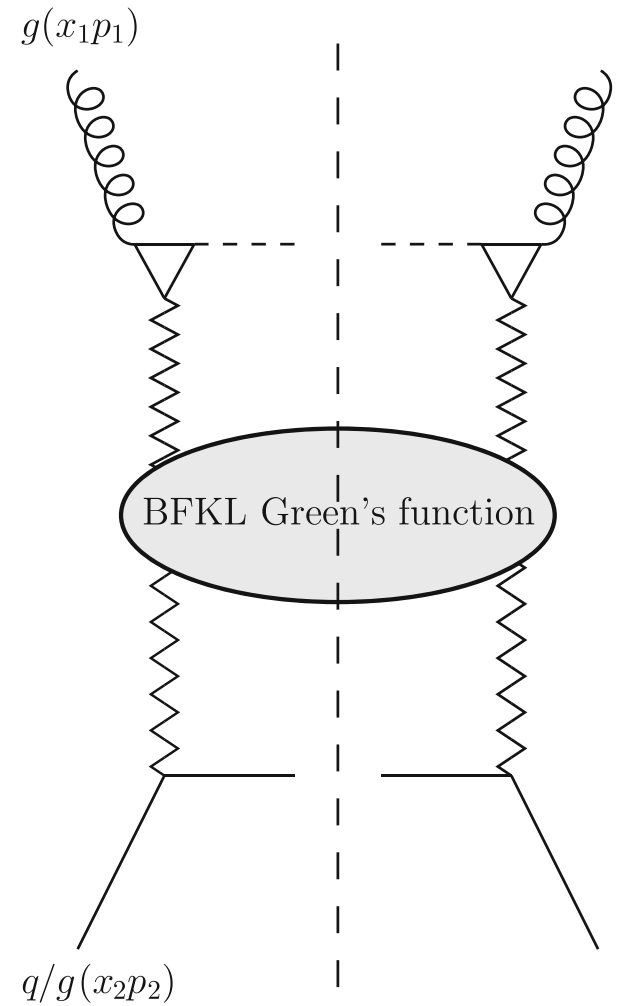

Fig. 2 Schematic representation of the BFKL factorization for the Higgs-jet hadroproduction

\section{Theoretical framework}

For the process under consideration (see Fig. 1) we plan to construct the cross section, differential in some of the kinematic variables of the Higgs and the jet, and some azimuthal correlations between them. In the BFKL approach the cross section takes the factorized form, diagrammatically represented in Fig. 2, given by the convolution of the Higgs and jet impact factors with the BFKL gluon Green's function, $G$.

\subsection{Forward-Higgs LO impact factor}

We can define the LO impact factor for the production of the Higgs in the gluon-gluon fusion channel, as follows (see, e.g., Ref. [122]):

$V_{g \rightarrow H}^{(0)}(\vec{q})=\sum_{\{f\}} \int \frac{d s_{g R}}{2 \pi} d(\mathrm{PS})^{(f)}|\mathcal{M}|^{2}$,

where $\mathcal{M}$ is the amplitude for the scattering of a gluon $g$, emitted by the colliding proton, off a Reggeon $R$ to produce a final state $f$, which at the LO, can only consist in a Higgs particle (see Fig. 3 for a representation of $|\mathcal{M}|^{2}$ ). The integration over the phase space $d(\mathrm{PS})^{(f)}$ then simply gives

$\mathrm{PS}^{(1)}=\int \frac{d^{4} p_{H}}{(2 \pi)^{4}}(2 \pi) \delta\left(p_{H}^{2}-M_{H}^{2}\right)(2 \pi)^{4} \delta^{(4)}\left(k+q-p_{H}\right)$ 


$$
=(2 \pi) \delta\left(s_{g R}-M_{H}^{2}\right),
$$

where $p_{H}$ is the Higgs boson momentum. Using this result, we end up with

$V_{g \rightarrow H}^{(0)}(\vec{q})=\frac{\alpha_{s}^{2}}{v^{2}} \frac{\vec{q}^{2}\left|\mathcal{F}\left(\vec{q}^{2}\right)\right|^{2}}{128 \pi^{2} \sqrt{N_{c}^{2}-1}}$,

with $\left.V_{g \rightarrow H}^{(0)}(\vec{q})\right|_{\vec{q}=0} \rightarrow 0$, so that the infra-red finiteness of the BFKL amplitude is preserved. Here $v$ is the electroweak vacuum expectation value parameter, $v^{2}=1 /\left(G_{F} \sqrt{2}\right)$, and

$$
\mathcal{F}\left(\vec{q}^{2}\right)=4 \int_{0}^{1} d y \int_{0}^{1-y} d x \frac{1-4 x y}{1-\left(\frac{M_{H, \perp}^{2}}{M_{t}^{2}}\right) x y+\left(\frac{\vec{q}^{2}}{M_{t}^{2}}\right) y(1-y)} .
$$

In this way we confirm, up to an irrelevant sign for $\mathcal{F}\left(\vec{q}^{2}\right)$, the results obtained earlier in Ref. [123]:

$$
\begin{aligned}
\mathcal{F}\left(\vec{q}^{2}\right)= & \frac{-4 M_{t}^{2}}{M_{H, \perp}^{2}}\left\{-2-\left(\frac{2 \vec{q}^{2}}{M_{H, \perp}^{2}}\right)\left[\sqrt{z_{1}} \mathcal{W}\left(z_{1}\right)-\sqrt{z_{2}} \mathcal{W}\left(z_{2}\right)\right]\right. \\
& \left.+\frac{1}{2}\left(1-\frac{4 M_{t}^{2}}{M_{H, \perp}^{2}}\right)\left[\mathcal{W}\left(z_{1}\right)^{2}-\mathcal{W}\left(z_{2}\right)^{2}\right]\right\}
\end{aligned}
$$

with $\vec{q}$ the transverse component of the four-vector $q$, $M_{H, \perp}=\sqrt{M_{H}^{2}+|\vec{q}|^{2}}$ the Higgs-boson transverse mass, $z_{1}=1-4 M_{t}^{2} / M_{H}^{2}, z_{2}=1+4 M_{t}^{2} / \vec{q}^{2}$, and the root $\sqrt{z_{1}}=i \sqrt{\left|z_{1}\right|}$ is taken for negative values of $z_{1}$. Furthermore, we have

$\mathcal{W}(z)= \begin{cases}-2 i \arcsin \frac{1}{\sqrt{1-z}}, & z<0 ; \\ \ln \frac{1+\sqrt{z}}{1-\sqrt{z}}-i \pi, & 0<z<1 ; \\ \ln \frac{1+\sqrt{z}}{\sqrt{z}-1}, & z>1 .\end{cases}$

In the large top-mass limit, our LO impact factor reads

$V_{g \rightarrow H}^{(0)}(\vec{q})=\frac{\alpha_{s}^{2}}{v^{2}} \frac{\vec{q}^{2}}{72 \pi^{2} \sqrt{N_{c}^{2}-1}}$.

The inclusion of the gluon PDF allows one to write differential proton-to-Higgs IF

$d V_{p \rightarrow H}^{(0)}(\vec{q})=\frac{\alpha_{s}^{2}}{v^{2}} \frac{\left|\mathcal{F}\left(\vec{q}^{2}\right)\right|^{2}}{128 \pi^{2} \sqrt{N_{c}^{2}-1}} \vec{q}^{2} d x_{H} f_{g}\left(x_{H}\right)$,

where the subscript $p$ in the left-hand-side denotes now the proton, $d x_{H}$ stands for the gluon/Higgs longitudinal momentum fraction. In order to establish the proper normalization for our impact factor, we insert into (8) a delta function depending on the produced Higgs-boson transverse momentum $\vec{p}_{H}$, then the LO result for the impact factor reads

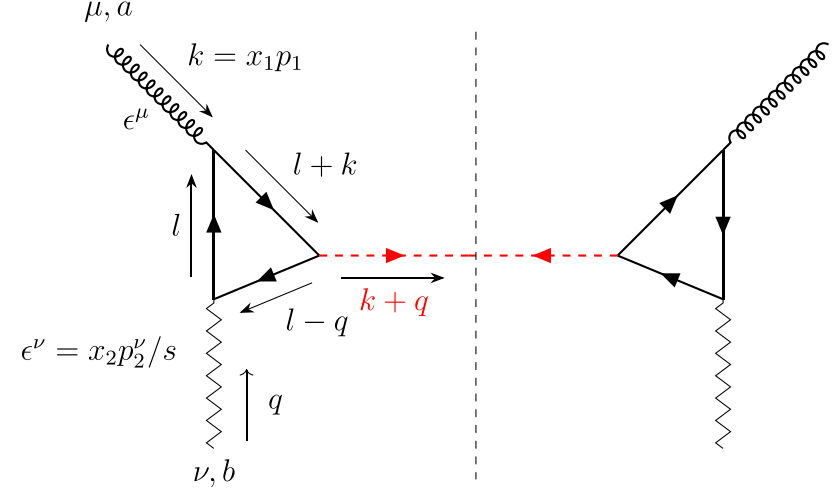

Fig. 3 Representative Feynman diagram for the squared modulus of the amplitude for the gluon scattering off a Reggeon to produce a Higgs particle. The Reggeized gluon is depicted by the zigzag line

$$
\begin{aligned}
& \frac{d V_{p \rightarrow H}^{(0)}(\vec{q})}{\vec{q}^{2}}=\frac{\alpha_{s}^{2}}{v^{2}} \frac{\left|\mathcal{F}\left(\vec{q}^{2}\right)\right|^{2}}{128 \pi^{2} \sqrt{N_{c}^{2}-1}} \vec{q}^{2} \\
& \quad \times \int_{0}^{1} d x_{H} f_{g}\left(x_{H}\right) \frac{d^{2} \vec{p}_{H}}{\vec{p}_{H}^{2}} \delta^{(2)}\left(\vec{p}_{H}-\vec{q}\right) .
\end{aligned}
$$

For later convenience, we transfer the impact factor to the so called $(v, n)$-representation, i.e. we express it as superposition of the eigenfunctions of LO BFKL kernel. The outcome is the following:

$d V_{p \rightarrow H}^{(0)}(\nu, n)=\int d^{2} \vec{q} \frac{d V_{p \rightarrow H}^{(0)}(\vec{q})}{\vec{q}^{2}} \frac{\left(\vec{q}^{2}\right)^{i \nu-1 / 2}}{\pi \sqrt{2}} e^{i n \phi}$,

where $\phi$ is the azimuthal angle of the vector $\vec{q}$. Combining Eqs. (9) and (10), we get the following differential expression for our LO impact factor:

$$
\begin{aligned}
& \frac{d V_{p \rightarrow H}^{(0)}(v, n)}{d x_{H} d^{2} \vec{p}_{H}} \\
& =\frac{\alpha_{s}^{2}}{v^{2}} \frac{\left|\mathcal{F}\left(\vec{p}_{H}^{2}\right)\right|^{2}}{128 \pi^{3} \sqrt{2\left(N_{c}^{2}-1\right)}}\left(\vec{p}_{H}^{2}\right)^{i v-1 / 2} f_{g}\left(x_{H}\right) e^{i n \phi_{H}},
\end{aligned}
$$

where $\phi_{H}$ denotes the azimuthal angle of the vector $\overrightarrow{p_{H}}$.

For the sake of completeness, we give the corresponding expression for the jet LO impact factor [21]

$$
\begin{aligned}
& \frac{d \Phi_{J}^{(0)}(\nu, n)}{d x_{J} d^{2} \vec{p}_{J}} \\
& =2 \alpha_{S} \sqrt{\frac{C_{F}}{C_{A}}}\left(\vec{p}_{J}^{2}\right)^{i \nu-3 / 2}\left(\frac{C_{A}}{C_{F}} f_{g}\left(x_{J}\right)+\sum_{a=q \bar{q}} f_{a}\left(x_{J}\right)\right) e^{i n \phi_{J}},
\end{aligned}
$$


where $\phi_{J}$ denotes the azimuthal angle of the vector $\vec{p}_{J}$.

In the next section we will build the cross section for the process of our consideration, by combining the BFKL Green's function and impact factor for the jet, together with our calculated Higgs-gluon impact factor.

\subsection{Cross section and azimuthal coefficients}

For the sake of simplicity, we consider final-state configurations where the Higgs is always tagged in a more forward direction with respect to the jet, thus implying $\Delta Y \equiv$ $y_{H}-y_{J}>0$.

As anticipated, the Higgs and the jet are also expected to feature large transverse momenta, $\left|\vec{p}_{H}\right|^{2} \sim\left|\vec{p}_{J}\right|^{2} \gg \Lambda_{\mathrm{QCD}}^{2}$. The four-momenta of the parent protons, $p_{1,2}$, are taken as Sudakov vectors satisfying $p_{1,2}^{2}=0$ and $p_{1} p_{2}=s / 2$, so that the final-state transverse momenta can be decomposed in the following way:

$$
\begin{gathered}
p_{H}=x_{H} p_{1}+\frac{M_{H, \perp}^{2}}{x_{H} S} p_{2}+p_{H \perp}, \quad p_{H \perp}^{2}=-\left|\vec{p}_{H}\right|^{2}, \\
p_{J}=x_{J} p_{2}+\frac{\left|\vec{p}_{J}\right|^{2}}{x_{J} S} p_{1}+p_{J \perp}, \quad p_{J \perp}^{2}=-\left|\vec{p}_{J}\right|^{2},
\end{gathered}
$$

with the space part of the four-vector $p_{1 \|}$ being taken positive; $M_{H, \perp}=\sqrt{M_{H}^{2}+\left|\vec{p}_{H}\right|^{2}}$ is the Higgs-boson transverse mass.

The longitudinal-momentum fractions, $x_{H, J}$, for the Higgs and jet are related to the corresponding rapidities in the center-of-mass frame via the relations

$$
\begin{gathered}
y_{H}=\frac{1}{2} \ln \frac{x_{H}^{2} s}{M_{H, \perp}^{2}}, \quad y_{J}=\frac{1}{2} \ln \frac{\left|\vec{p}_{J}\right|^{2}}{x_{J}^{2} s}, \\
d y_{H, J}= \pm \frac{d x_{H, J}}{x_{H, J}} .
\end{gathered}
$$

$$
\begin{aligned}
& \mathcal{C}_{n} \equiv \int_{0}^{2 \pi} d \varphi_{H} \int_{0}^{2 \pi} d \varphi_{J} \cos (n \varphi) \frac{d \sigma}{d y_{H} d y_{J} d\left|\vec{p}_{H}\right| d\left|\vec{p}_{J}\right| d \varphi_{H} d \varphi_{J}}=\frac{e^{\Delta Y}}{s} \frac{M_{H, \perp}}{\left|\vec{p}_{H}\right|}
\end{aligned}
$$

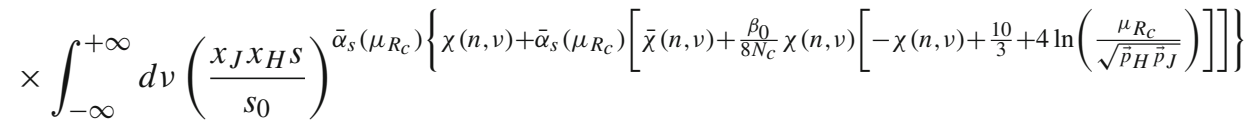

$$
\begin{aligned}
& \times\left\{\alpha_{s}^{2}\left(\mu_{R_{1}}\right) c_{H}\left(n, v,\left|\vec{p}_{H}\right|, x_{H}\right)\right\}\left\{\alpha_{S}\left(\mu_{R_{2}}\right)\left[c_{J}\left(n, v,\left|\vec{p}_{J}\right|, x_{J}\right)\right]^{*}\right\} \\
& \times\left\{1+\alpha_{s}\left(\mu_{R_{1}}\right) \frac{c_{H}^{(1)}\left(n, v,\left|\vec{p}_{H}\right|, x_{H}\right)}{c_{H}\left(n, v,\left|\vec{p}_{H}\right|, x_{H}\right)}+\alpha_{s}\left(\mu_{R_{2}}\right)\left[\frac{c_{J}^{(1)}\left(n, v,\left|\vec{p}_{J}\right|, x_{J}\right)}{c_{J}\left(n, v,\left|\vec{p}_{J}\right|, x_{J}\right)}\right]^{*}\right\},
\end{aligned}
$$

As for the rapidity distance, one has

$$
\Delta Y=y_{H}-y_{J}=\ln \frac{x_{H} x_{J} s}{M_{H, \perp}\left|\vec{p}_{J}\right|} .
$$

Using QCD collinear factorization to build the (differential) hadronic the cross section, one has

$$
\begin{aligned}
& \frac{d \sigma}{d x_{H} d x_{J} d^{2} \vec{p}_{H} d^{2} \vec{p}_{J}} \\
& =\sum_{i, j=q, \bar{q}, g} \int_{0}^{1} d x_{1} \int_{0}^{1} d x_{2} f_{i}\left(x_{1}, \mu_{F_{1}}\right) \\
& \quad \times f_{j}\left(x_{2}, \mu_{F_{2}}\right) \frac{d \hat{\sigma}_{i, j}\left(\hat{s}, \mu_{F_{1,2}}\right)}{d x_{H} d x_{J} d^{2} \vec{p}_{H} d^{2} \vec{p}_{J}},
\end{aligned}
$$

where the $i, j$ indices run over the parton kinds (quarks $q=u, d, s, c, b$; antiquarks $\bar{q}=\bar{u}, \bar{d}, \bar{s}, \bar{c}, \bar{b}$; or gluon $g$ ), $f_{i, j}\left(x, \mu_{F_{1,2}}\right)$ are the incoming-proton PDFs; $x_{1,2}$ denote the longitudinal fractions of the partons involved in the hard subprocess, whereas $\mu_{F_{1,2}}$ stand for the factorization scales characteristic of the two fragmentation regions of the incoming hadrons; $d \hat{\sigma}_{i, j}(\hat{s})$ is the partonic cross section, with $\hat{s} \equiv x_{1} x_{2} s$ the squared center-of-mass energy of the partonparton collision subreaction. In the present case, the sum over the parton kinds $i$ restricts to the gluon contribution only, consistently with a LO treatment of the Higgs impact factor, as discussed in the previous section.

The BFKL cross section can be presented (see Ref. [43] for the derivation) as the Fourier series of the so-called azimuthal coefficients, $\mathcal{C}_{n}$

$$
\begin{gathered}
\frac{d \sigma}{d y_{H} d y_{J} d\left|\vec{p}_{H}\right| d\left|\vec{p}_{J}\right| d \varphi_{H} d \varphi_{J}} \\
=\frac{1}{(2 \pi)^{2}}\left[\mathcal{C}_{0}+\sum_{n=1}^{\infty} 2 \cos (n \varphi) \mathcal{C}_{n}\right]
\end{gathered}
$$

where $\varphi=\varphi_{H}-\varphi_{J}-\pi$, with $\varphi_{H, J}$ the Higgs and the jet azimuthal angles. A comprehensive formula for the $\varphi$ averaged cross section, $\mathcal{C}_{0}$, and the other coefficients, $\mathcal{C}_{n>0}$, reads 
the first coefficient in the expansion of the QCD $\beta$-function ( $n_{f}$ is the active-flavor number),

$\chi(n, v)=2 \psi(1)-\psi\left(\frac{n+1}{2}+i v\right)-\psi\left(\frac{n+1}{2}-i v\right)$

the eigenvalue of the LO BFKL kernel, $c_{H, J}(n, v)$ are the Higgs and the jet LO impact factors in the $(v, n)$-space, given by

$$
\begin{aligned}
& c_{H}\left(n, v,\left|\vec{p}_{H}\right|, x_{H}\right) \\
& =\frac{1}{v^{2}} \frac{\left|\mathcal{F}\left(\vec{p}_{H}^{2}\right)\right|^{2}}{128 \pi^{3} \sqrt{2\left(N_{c}^{2}-1\right)}}\left(\vec{p}_{H}^{2}\right)^{i v+1 / 2} f_{g}\left(x_{H}, \mu_{F_{1}}\right), \\
& c_{J}\left(n, v,\left|\vec{p}_{J}\right|, x_{J}\right) \\
& =2 \sqrt{\frac{C_{F}}{C_{A}}}\left(\vec{p}_{J}^{2}\right)^{i \nu-1 / 2}\left(\frac{C_{A}}{C_{F}} f_{g}\left(x_{J}, \mu_{F_{2}}\right)\right. \\
& \left.\quad+\sum_{a=q, \bar{q}} f_{a}\left(x_{J}, \mu_{F_{2}}\right)\right) .
\end{aligned}
$$

The energy-scale parameter, $s_{0}$, is arbitrary within NLA accuracy and will be fixed in our analysis at $s_{0}=M_{H, \perp}\left|\vec{p}_{J}\right|$. The remaining quantities are the NLO impact-factor corrections, $c_{H, J}^{(1)}\left(n, v,\left|\vec{p}_{H, J}\right|, x_{H, J}\right)$. The expression for the Higgs NLO impact factor has not been yet calculated. It is possible, however, to include some "universal" NLO contributions to the Higgs impact factor, which can be expressed through the corresponding LO impact factor, and are fixed by the requirement of stability within the NLO under variations of the energy scale $s_{0}$, the renormalization scale $\mu_{R}$ and of the factorization scale $\mu_{F}$, getting

$\alpha_{s} c_{H}^{(1)}\left(n, v,\left|\vec{p}_{H}\right|, x_{H}\right) \rightarrow \bar{\alpha}_{s} \tilde{c}_{H}^{(1)}\left(n, v,\left|\vec{p}_{H}\right|, x_{H}\right)$,

with

$$
\begin{aligned}
& \tilde{c}_{H}^{(1)}\left(n, v,\left|\vec{p}_{H}\right|, x_{H}\right) \\
& =c_{H}\left(n, v,\left|\vec{p}_{H}\right|, x_{H}\right)\left\{\frac{\beta_{0}}{4 N_{c}}\left(2 \ln \frac{\mu_{R_{1}}}{\left|\vec{p}_{H}\right|}+\frac{5}{3}\right)\right. \\
& \quad+\frac{\chi(n, v)}{2} \ln \left(\frac{s_{0}}{M_{H, \perp}^{2}}\right)
\end{aligned}
$$

The jet impact factor is known at the NLO [18-22], and at that order it is not universal, since it depends on the adopted jet selection function (see, e.g., Ref. [22]). Nonetheless we treated it on the same ground as the Higgs one, including only the NLO corrections fixed by the renormalization group and leading to

$$
\begin{aligned}
\tilde{c}_{J}^{(1)}\left(n, v,\left|\vec{p}_{J}\right|, x_{J}\right) \\
=c_{J}\left(n, v,\left|\vec{p}_{J}\right|, x_{J}\right)\left\{\frac{\beta_{0}}{4 N_{c}}\left(2 \ln \frac{\mu_{R_{2}}}{\left|\vec{p}_{J}\right|}+\frac{5}{3}\right)\right. \\
+\frac{\chi(n, v)}{2} \ln \left(\frac{s_{0}}{\left|\vec{p}_{J}\right|^{2}}\right) \\
-\frac{1}{2 N_{c}\left(\frac{C_{A}}{C_{F}} f_{g}\left(x_{J}, \mu_{F_{2}}\right)+\sum_{a=q, \bar{q}} f_{a}\left(x_{J}, \mu_{F_{2}}\right)\right)} \ln \frac{\mu_{F_{2}}^{2}}{\left|\vec{p}_{J}\right|^{2}} \\
\quad \times\left(\frac { C _ { A } } { C _ { F } } \int _ { x _ { J } } ^ { 1 } \frac { d z } { z } \left[P_{g g}(z) f_{g}\left(\frac{x_{J}}{z}, \mu_{F_{2}}\right)\right.\right. \\
\left.+\sum_{a=q, \bar{q}} P_{g a}(z) f_{a}\left(\frac{x_{J}}{z}, \mu_{F_{2}}\right)\right] \\
+\sum_{a=q, \bar{q}} \int_{x_{J}}^{1} \frac{d z}{z}\left[P_{a g}(z) f_{g}\left(\frac{x_{J}}{z}, \mu_{F_{2}}\right)\right. \\
\left.\left.\left.+P_{a a}(z) f_{a}\left(\frac{x_{J}}{z}, \mu_{F_{2}}\right)\right]\right)\right\} .
\end{aligned}
$$

Combining all the ingredients, we can write our master formula for the azimuthal coefficients, 


$$
\begin{aligned}
& \mathcal{C}_{n}=\frac{e^{\Delta Y}}{s} \frac{M_{H, \perp}}{\left|\vec{p}_{H}\right|}
\end{aligned}
$$

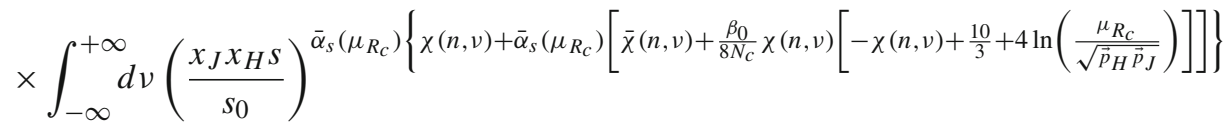

$$
\begin{aligned}
& \times\left\{\alpha_{s}^{2}\left(\mu_{R_{1}}\right) c_{H}\left(n, v,\left|\vec{p}_{H}\right|, x_{H}\right)\right\}\left\{\alpha_{S}\left(\mu_{R_{2}}\right)\left[c_{J}\left(n, v,\left|\vec{p}_{J}\right|, x_{J}\right)\right]^{*}\right\} \\
& \times\left\{1+\bar{\alpha}_{S}\left(\mu_{R_{1}}\right) \frac{\tilde{c}_{H}^{(1)}\left(n, v,\left|\vec{p}_{H}\right|, x_{H}\right)}{c_{H}\left(n, v,\left|\vec{p}_{H}\right|, x_{H}\right)}+\bar{\alpha}_{s}\left(\mu_{R_{2}}\right)\left[\frac{\tilde{c}_{J}^{(1)}\left(n, v,\left|\vec{p}_{J}\right|, x_{J}\right)}{c_{J}\left(n, v,\left|\vec{p}_{J}\right|, x_{J}\right)}\right]^{*}\right\} .
\end{aligned}
$$

The renormalization scales $\left(\mu_{R_{1,2, c}}\right)$ and the factorization ones $\left(\mu_{F_{1,2}}\right)$ can, in principle, be chosen arbitrarily, since their variation produces effects beyond the NLO. It is however advisable to relate them to the physical hard scales of the process. We chose to fix them differently from each other, depending on the subprocess to which they are related: $\mu_{R_{1}} \equiv \mu_{F_{1}}=C_{\mu} M_{H, \perp}, \mu_{R_{2}} \equiv \mu_{F_{2}}=C_{\mu}\left|\vec{p}_{J}\right|, \mu_{R_{c}}=$ $C_{\mu} \sqrt{M_{H, \perp}\left|\vec{p}_{J}\right|}$, where $C_{\mu}$ is a variation parameter introduced to gauge the effect of a change of the scale (see the discussion at the end of Sect. 3.2).

\section{Phenomenology}

\subsection{Azimuthal correlations and $p_{T}$-distribution}

The first observables of our consideration are the azimuthalangle coefficients integrated over the phase space for two final-state particles, while the rapidity interval, $\Delta Y$, between the Higgs boson and the jet is kept fixed:

$$
\begin{aligned}
& C_{n}(\Delta Y, s) \\
& =\int_{p_{H}^{\min }}^{p_{H}^{\max }} d\left|\vec{p}_{H}\right| \int_{p_{J}^{\min }}^{p_{J}^{\max }} d\left|\vec{p}_{J}\right| \int_{y_{H}^{\min }}^{y_{H}^{\max }} d y_{H} \\
& \quad \times \int_{y_{J}^{\min }}^{y_{J}^{\max }} d y_{J} \delta\left(y_{H}-y_{J}-\Delta Y\right) \mathcal{C}_{n} .
\end{aligned}
$$

Pursuing the goal of fitting realistic kinematic cuts adopted by the current experimental analyses at the LHC, we constrain the Higgs emission inside the rapidity acceptances of the CMS barrel detector, i.e. $\left|y_{H}\right|<2.5$, while we allow for a larger rapidity range of the jet [125], which can be detected also by the CMS endcaps, namely $\left|y_{J}\right|<4$.7. Furthermore, three distinct cases for the final-state transverse momenta are considered:

(a) symmetric configuration, suited to the search of pure BFKL effects, where both the Higgs and the jet transverse momenta lie in the range: $20 \mathrm{GeV}<\left|\vec{p}_{H, J}\right|<$ $60 \mathrm{GeV}$;

(b) asymmetric selection, typical of the ongoing LHC phenomenology, where the Higgs transverse momentum runs from $10 \mathrm{GeV}$ to $2 M_{t}$, where the jet is tagged inside its typical CMS configuration, from 20 to $60 \mathrm{GeV}$;

(c) disjoint windows, which allows for the maximum exclusiveness in the final state: $35 \mathrm{GeV}<\left|\vec{p}_{J}\right|<60 \mathrm{GeV}$ and $60 \mathrm{GeV}<\left|\vec{p}_{H}\right|<2 M_{t}$.

We study the $\varphi$-averaged cross section (alias the $\Delta Y$ distribution), $C_{0}(\Delta Y, s)$, the azimuthal-correlation moments, $R_{n 0}(\Delta Y, s)=C_{n} / C_{0} \equiv\langle\cos n \varphi\rangle$, and their ratios, $R_{n m}=$ $C_{n} / C_{m}[113,114]$ as functions of the Higgs-jet rapidity distance, $\Delta Y$.

The second observable of our interest is the $p_{H}$-distribution for a given value of $\Delta Y$ :

$$
\begin{aligned}
& \frac{d \sigma\left(\left|\vec{p}_{H}\right|, \Delta Y, s\right)}{d\left|\vec{p}_{H}\right| d \Delta Y} \\
& =\int_{p_{J}^{\min }}^{p_{J}^{\max }} d\left|\vec{p}_{J}\right| \int_{y_{H}^{\min }}^{y_{H}^{\max }} d y_{H} \int_{y_{J}^{\min }}^{y_{J}^{\max }} d y_{J} \\
& \quad \delta\left(y_{H}-y_{J}-\Delta Y\right) \mathcal{C}_{0},
\end{aligned}
$$

the Higgs and jet rapidity ranges being given above and $35 \mathrm{GeV}<\left|\vec{p}_{J}\right|<60 \mathrm{GeV}$.

\subsection{Results and discussion}

In Fig. 4 we present results for the $\Delta Y$-distribution, $C_{0}$, in the three kinematic configurations under investigation. Here, the usual onset of the BFKL dynamics comes easily out. The growth with energy of the pure partonic cross sections is quenched by the convolution with PDFs, this leading to a lowering with $\Delta Y$ of hadronic distributions. Notably, NLA predictions (red) are almost entirely contained inside LLA uncertainty bands (blue), thus corroborating the underlying assumption that the large energy scales provided by the emission of a Higgs boson stabilize the BFKL series. A further manifestation of this effect appears in the analysis of azimuthal correlations, $R_{n m}$. For all the considered cases (Figs. 5, 6 and 7), higher-order corrections show a milder discrepancy with respect to pure LLA ones. This represents a novel feature in the context of semi-hard reactions, where LLA moments have always shown a fairly stronger decorrelation than NLA ones. Previous studies of Mueller-Navelet jet production $[45,48,50]$ have highlighted how the use of scale- 
optimization procedures is needed to bring NLA patterns near LLA ones and, ultimately, to match CMS data [125]. Conversely, Higgs-jet hadroproduction genuinely exhibits a solid stability under higher-order corrections in the range between $1 / 2$ and two times the natural scales provided by kinematics, thus tracing the path towards possible precision studies of cross sections. We limit ourselves to the calculation of azimuthal coefficients up to $n=3$. The extension of our study to higher azimuthal coefficients makes possible to construct other observables, such as angular distributions in $\cos \varphi$, as suggested in Ref. [126].

In Fig. 8 we present predictions for the $p_{H}$-distribution, $d \sigma /\left(d\left|\vec{p}_{H}\right| d \Delta Y\right)$, in the range $10 \mathrm{GeV}<\left|\vec{p}_{H}\right|<2 M_{t}$, and for two values of the rapidity interval, $\Delta Y=3,5$. Here, the Born contribution (green) corresponds to the so-called two-gluon approximation, which describes the back-to-back emission of the Higgs and of the jet with no additional gluon radiation. From the analytic point of view, one has

$$
\begin{aligned}
& \frac{d \sigma^{\text {Born }}\left(\left|\vec{p}_{H}\right|, \Delta Y, s\right)}{d\left|\vec{p}_{H}\right| d \Delta Y} \\
& =\pi \frac{e^{\Delta Y}}{s} M_{H, \perp} \int_{y_{H}^{\min }}^{y_{H}^{\max }} d y_{H} \int_{y_{J}^{\min }}^{y_{J}^{\max }} d y_{J} \delta\left(y_{H}-y_{J}-\Delta Y\right) \\
& \quad \times \alpha_{s}^{2}\left(\mu_{R_{1}}\right) \frac{1}{v^{2}} \frac{\left|\mathcal{F}\left(\vec{p}_{H}^{2}\right)\right|^{2}}{128 \pi^{3} \sqrt{2\left(N_{c}^{2}-1\right)}} f_{g}\left(x_{H}, \mu_{F_{1}}\right) \\
& \quad \times \alpha_{s}\left(\mu_{R_{2}}\right) 2 \sqrt{\frac{C_{F}}{C_{A}}}\left(\frac{C_{A}}{C_{F}} f_{g}\left(x_{J}, \mu_{F_{2}}\right)+\sum_{a=q, \bar{q}} f_{a}\left(x_{J}, \mu_{F_{2}}\right)\right) .
\end{aligned}
$$

Our calculation in the Born limit at $\Delta Y=3$ (left panel of Fig. 8) is in fair agreement with the corresponding pattern in Ref. [123] (solid line in the left panel of Fig. 2), up to a factor two, due to the fact that we restricted $\Delta Y$ to be positive, which means that the Higgs particle is always more forward than the jet. ${ }^{3}$ In our study, this calculation cannot exceed a given upper cut-off in the $\left|\vec{p}_{H}\right|$-range, say around $125 \mathrm{GeV}$. This is due to our choice for the final-state kinematic ranges, where consistency with experimental cuts in the rapidities of the detected objects would lead to $x_{J}>1$ for sufficiently large jet transverse momenta.

Both the LLA (blue) and the NLA (red) series of Fig. 8 show a peak (not present in the Born case) at $\left|\vec{p}_{H}\right|$ around $40 \mathrm{GeV}$ for the two values of $\Delta Y$, and a decreasing behavior at large $\left|\vec{p}_{H}\right|$. For the sake of simplicity, we distinguish three kinematic subregions. The low- $\left|\vec{p}_{H}\right|$ region, i.e. $\left|\vec{p}_{H}\right|<$ $10 \mathrm{GeV}$, has been excluded from our analysis, since it is dominated by large transverse-momentum logarithms, which call for the corresponding all-order resummation [127-143], not accounted by our formalism. To the intermediate- $\left|\vec{p}_{H}\right|$ region

\footnotetext{
${ }^{3}$ Note that in Ref. [123] the Higgs mass is a free parameter. We compare our result with the corresponding one at $M_{H}=120 \mathrm{GeV}$.
}

the set of configurations where $\left|\vec{p}_{H}\right|$ is of the same order of $\left|\vec{p}_{J}\right|$, which ranges from 35 to $60 \mathrm{GeV}$, corresponds. It is essentially the peak region plus the first part of the decreasing tail, where NLA bands are totally nested inside the LLA ones. Here, the impressive stability of the perturbative series unambiguously confirms the validity of our description at the hand of the BFKL resummation. Finally, in the large- $\left|\vec{p}_{H}\right|$ region represented by the long tail, NLA distributions decouple from LLA ones and exhibit an increasing sensitivity to scale variation. Here, DGLAP-type logarithms together with threshold effects [144-146] start to become relevant, thus spoiling the convergence of the high-energy series. In Fig. 8 we present also the $p_{H}$-distributions at $\Delta Y=3$ and 5 , as obtained by a fixed-order NLO calculation through the POWHEG method [147-149], by suitably adapting the subroutines dedicated to the inclusive Higgs plus jet final state $[150,151]$. It is interesting to observe that, both at $\Delta Y=3$ and $\Delta Y=5$, the NLO fixed-order prediction is systematically lower than the LLA- and NLA-BFKL ones and this is more evident at the larger $\Delta Y$, where the effect of resummation is expected to be more important. This observation provides with an interesting window for discrimination between fixed-order and high-energy-resummed approaches.

Finally, we compared the distributions presented above with the corresponding ones obtained in the large top-mass limit, $M_{t} \rightarrow+\infty$ (see Eq. 7). We noted that, when this limit is taken, cross sections become at most $5 \div 7 \%$ larger, whereas the effect on azimuthal correlations is very small or negligible. We do not show figures related with this comparison, since the bands related to the large top-mass limit are hardly distinguishable from the ones with physical top mass. The impact on the $p_{H}$-distribution (Fig. 9) is also quite small in the $\left|\vec{p}_{H}\right| \sim\left|\vec{p}_{J}\right|$ range, while it become more manifest when the value of $\left|\vec{p}_{H}\right|$ increases.

All these considerations brace the message that an exhaustive study of the $\left|\vec{p}_{H}\right|$-distribution would rely on a unified formalism where distinct resummations are concurrently embodied. In particular, the impact of the BFKL resummation could depend on the delicate interplay among the Higgs transverse mass, the Higgs transverse momentum and the jet transverse momentum entering, in logarithmic form, the expressions of partial NLO corrections to impact factors (see Eqs. (24) and (25)). Future studies including full higher-order corrections will allow us to further gauge the stability of our calculations.

\subsection{Numerical specifics and uncertainty estimation}

All the numerical studies were completed making use of JETHAD, a FORTRAN2008- PYTHON3 hybrid library under development at our Group, which has been recently employed in the analysis of hadron-jet correlations [71] and of the inclusive heavy-flavored jet-pair hadroproduction [79]. An aux- 


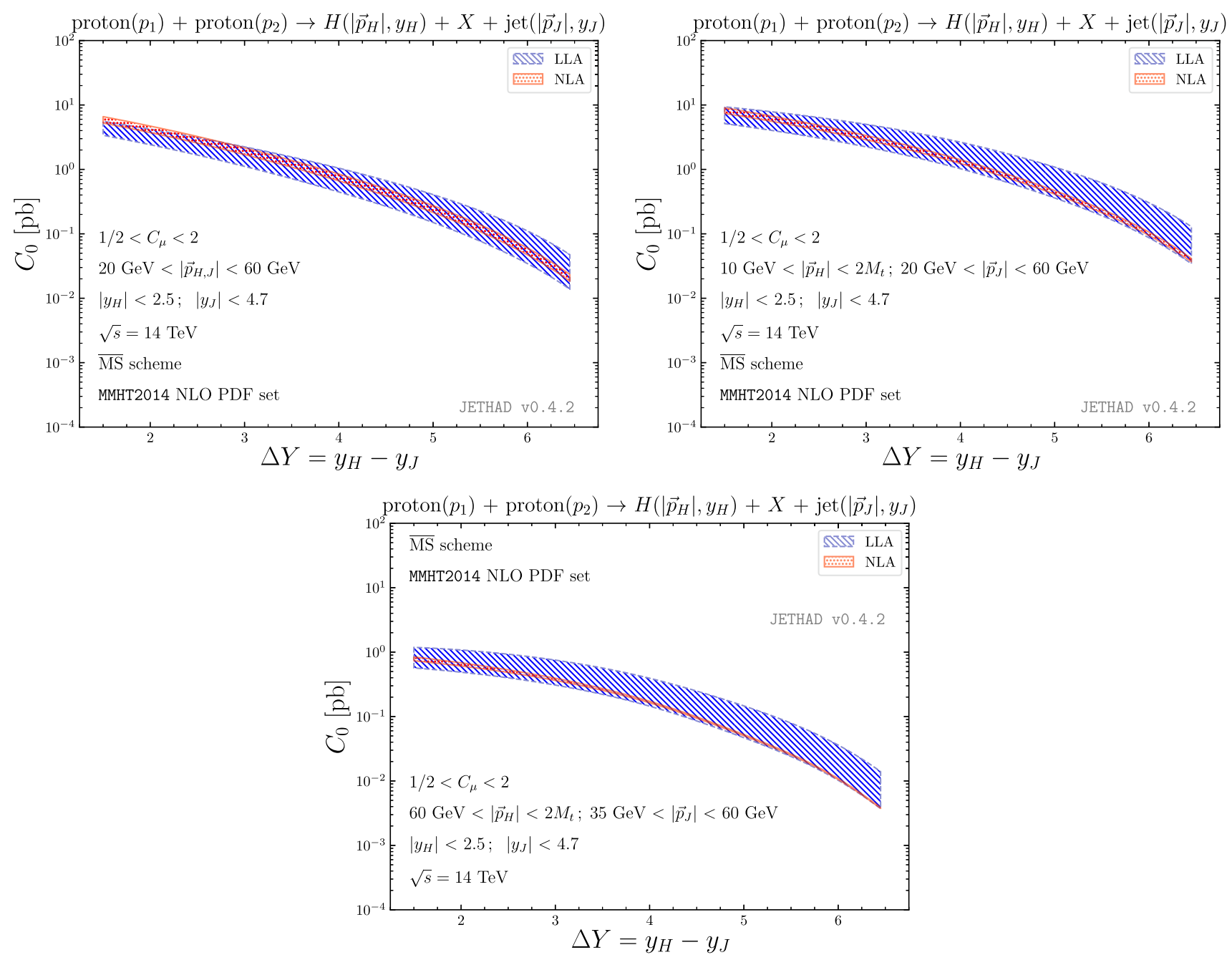

Fig. $4 \Delta Y$-dependence of the $\varphi$-averaged cross section, $C_{0}$, for the inclusive Higgs-jet hadroproduction in the three considered $p_{T}$-ranges and for $\sqrt{s}=14 \mathrm{TeV}$. Shaded bands give the combined effect of the scale variation with the uncertainty coming from the phase-space numerical integration

iliary, independent MATHEMATICA interface allowed us to test the numerical reliability of our results. Quark and gluon PDFs were calculated through the MMHT2014 NLO PDF set [152] as provided by the LHAPDFv6.2.1 interpolator [153], whereas we selected a two-loop running coupling setup with $\alpha_{s}\left(M_{Z}\right)=0.11707$ and with dynamic-flavor threshold.

The two relevant sources of numerical uncertainty respectively come from the multidimensional integration over the final-state phase space (together with the oscillatory $v$ distribution) and from the one-dimensional integral over the longitudinal momentum fraction $\zeta$ in the NLO impact factor corrections (Eqs. (24) and (25)). They were directly estimated by the JETHAD integration tools. Other potential uncertainties, as the upper cutoff in the numerical integrations over $\left|\vec{p}_{H}\right|,\left|\vec{p}_{J}\right|$ and the $v$-variable, turned out to be negligible with respect to the first ones.
Furthermore, we gauged the effect of concurrently varying the renormalization scales $\left(\mu_{R_{1,2, c}}\right)$ and the factorization ones $\left(\mu_{F_{1,2}}\right)$ of them around their natural values in the range 1/2 to two. The parameter $C_{\mu}$ entering the inset of panels in Figs. 4, 5, 6, 7 and 8 gives the ratio

$C_{\mu}=\frac{\mu_{R, F_{1}}}{M_{H, \perp}}=\frac{\mu_{R, F_{2}}}{\left|\vec{p}_{J}\right|}=\frac{\mu_{R_{c}}}{\sqrt{M_{H, \perp}\left|\vec{p}_{J}\right|}}$.

\section{Conclusions and outlook}

We have proposed the inclusive hadroproduction of a Higgs boson and of a jet featuring high transverse momenta and separated by a large rapidity distance as a new diffractive semihard channel to probe the BFKL resummation. Statistics for cross sections differential in rapidity, tailored on different configurations for transverse-momentum ranges at CMS, is 

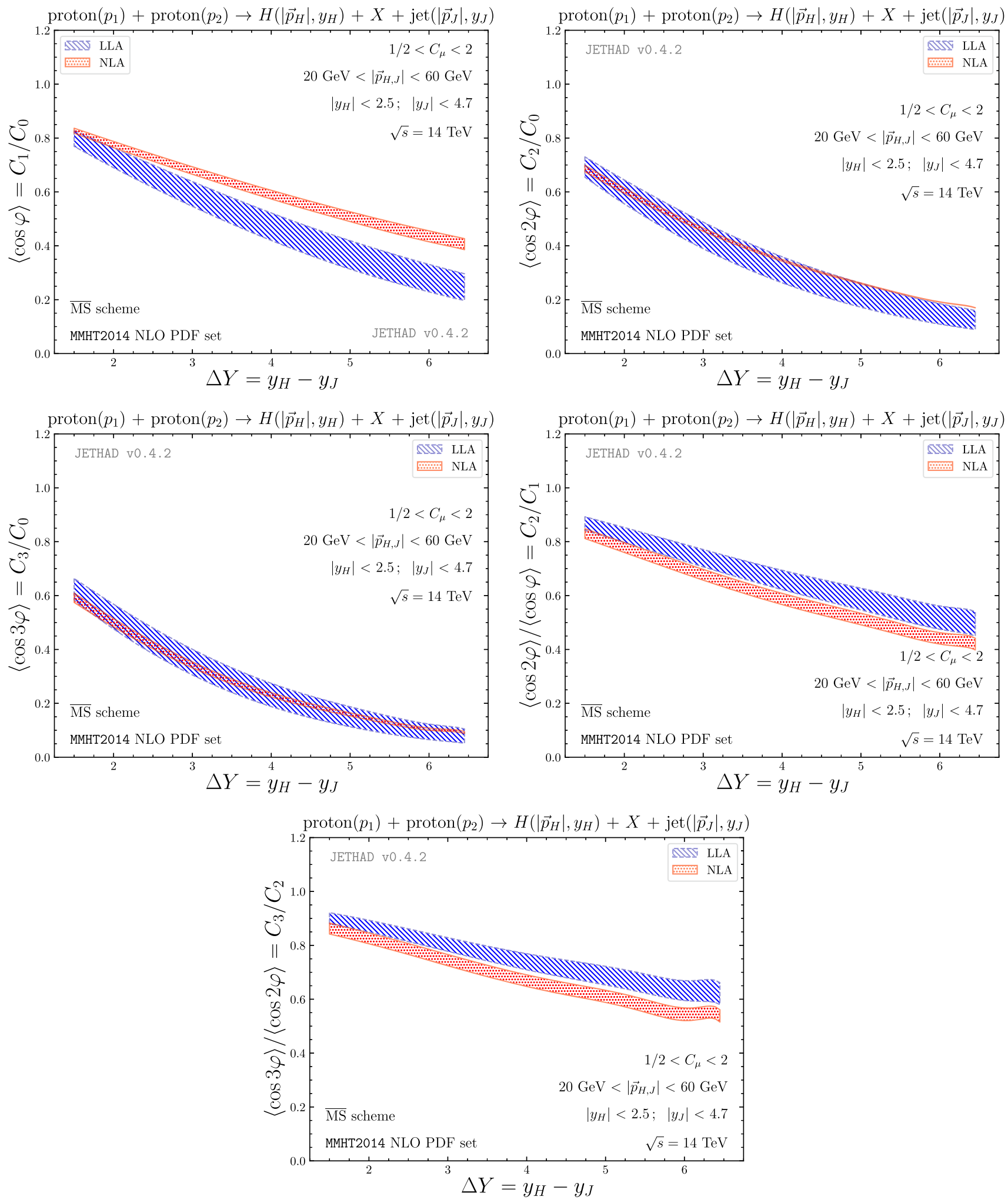

Fig. $5 \Delta Y$-dependence of several ratios $R_{n m} \equiv C_{n} / C_{m}$, for the inclusive Higgs-jet hadroproduction in the $p_{T}$-symmetric configuration and for $\sqrt{s}=14 \mathrm{TeV}$. Shaded bands give the combined effect of the scale variation with the uncertainty coming from the phase-space numerical integration 

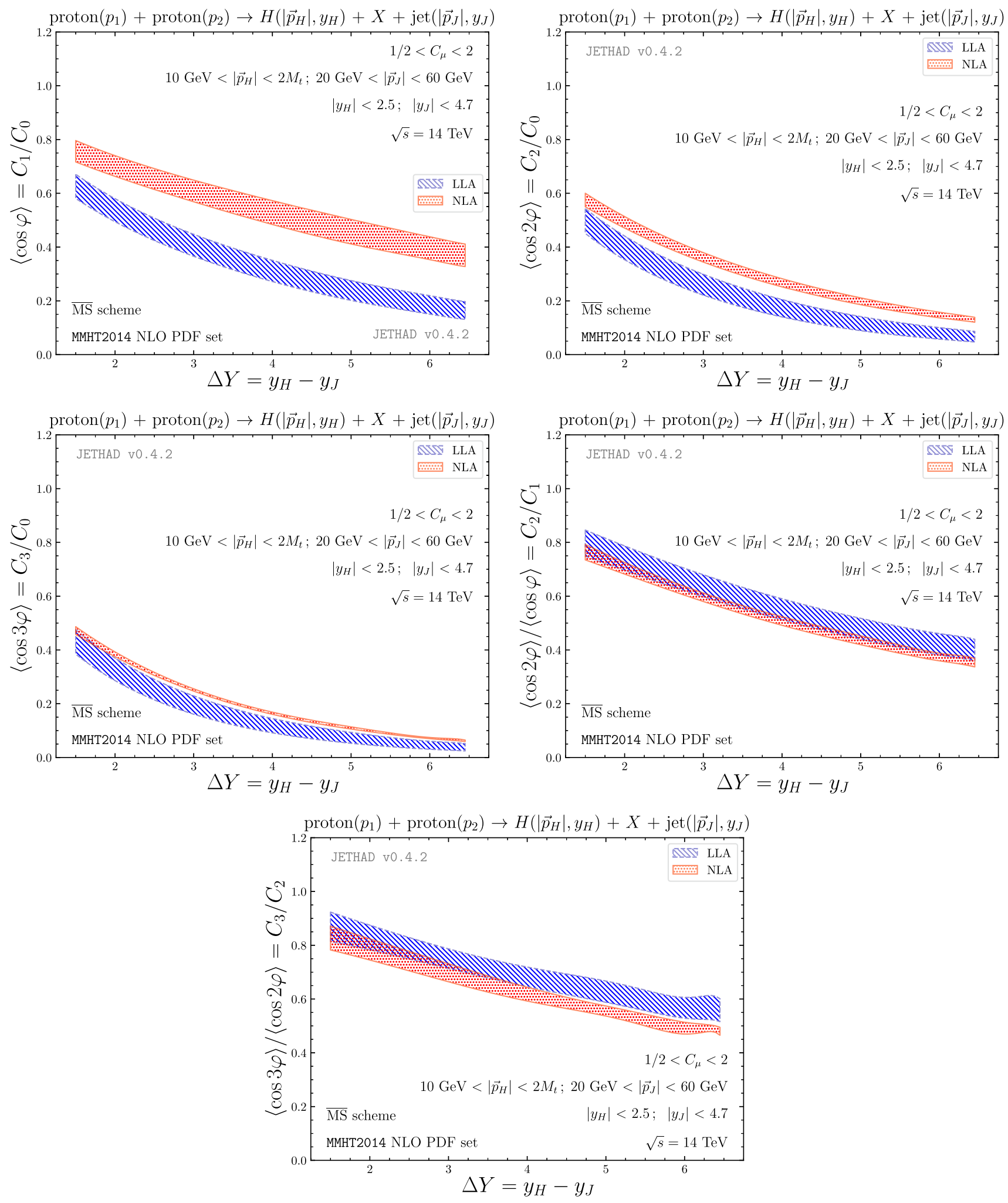

Fig. $6 \Delta Y$-dependence of several ratios $R_{n m} \equiv C_{n} / C_{m}$, for the inclusive Higgs-jet hadroproduction in the $p_{T}$-asymmetric configuration and for $\sqrt{s}=14 \mathrm{TeV}$. Shaded bands give the combined effect of the scale variation with the uncertainty coming from the phase-space numerical integration 

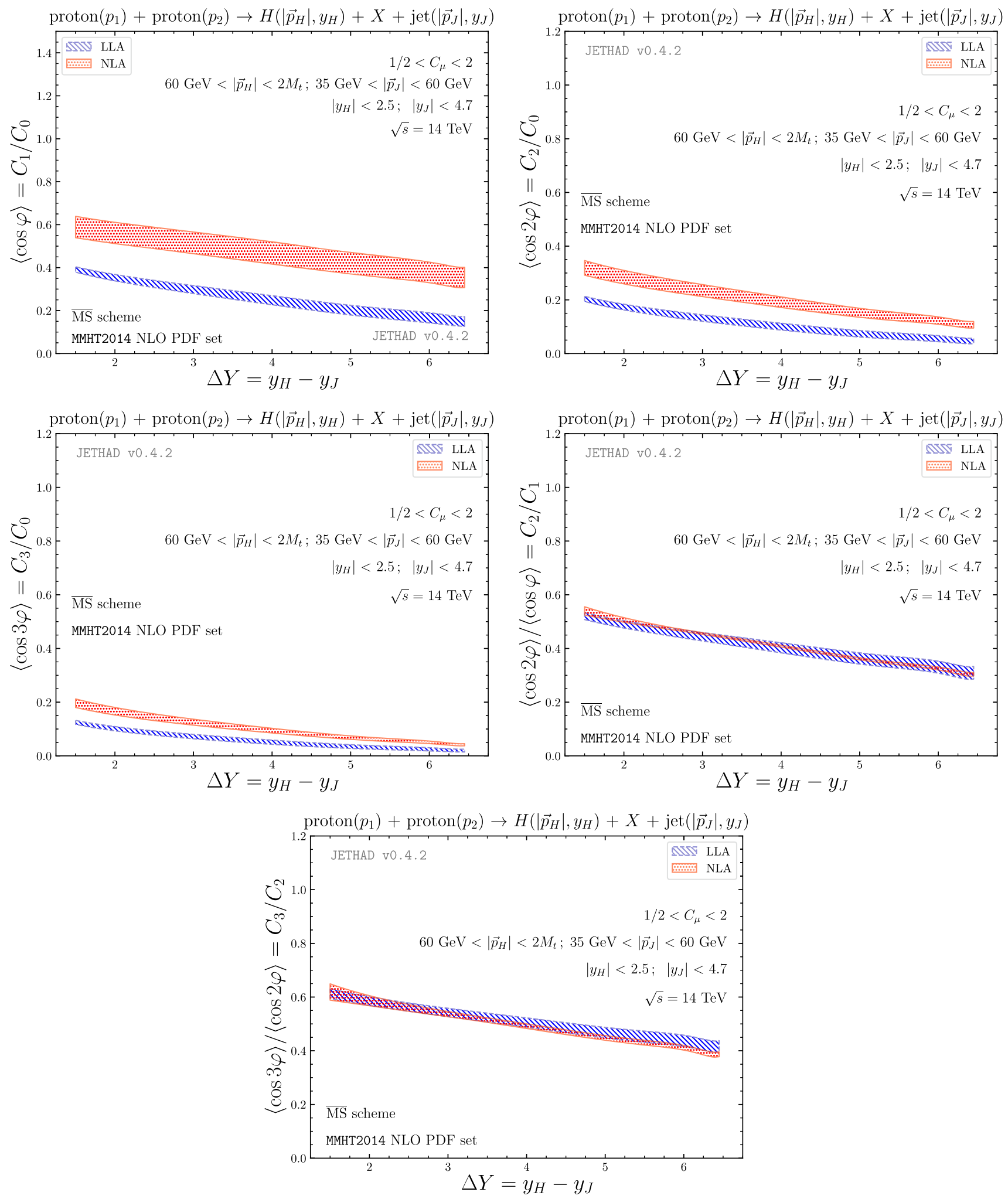

Fig. $7 \Delta Y$-dependence of several ratios $R_{n m} \equiv C_{n} / C_{m}$, for the inclusive Higgs-jet hadroproduction in the disjoint $p_{T}$-windows configuration and for $\sqrt{s}=14 \mathrm{TeV}$. Shaded bands give the combined effect of the scale variation with the uncertainty coming from the phase-space numerical integration 

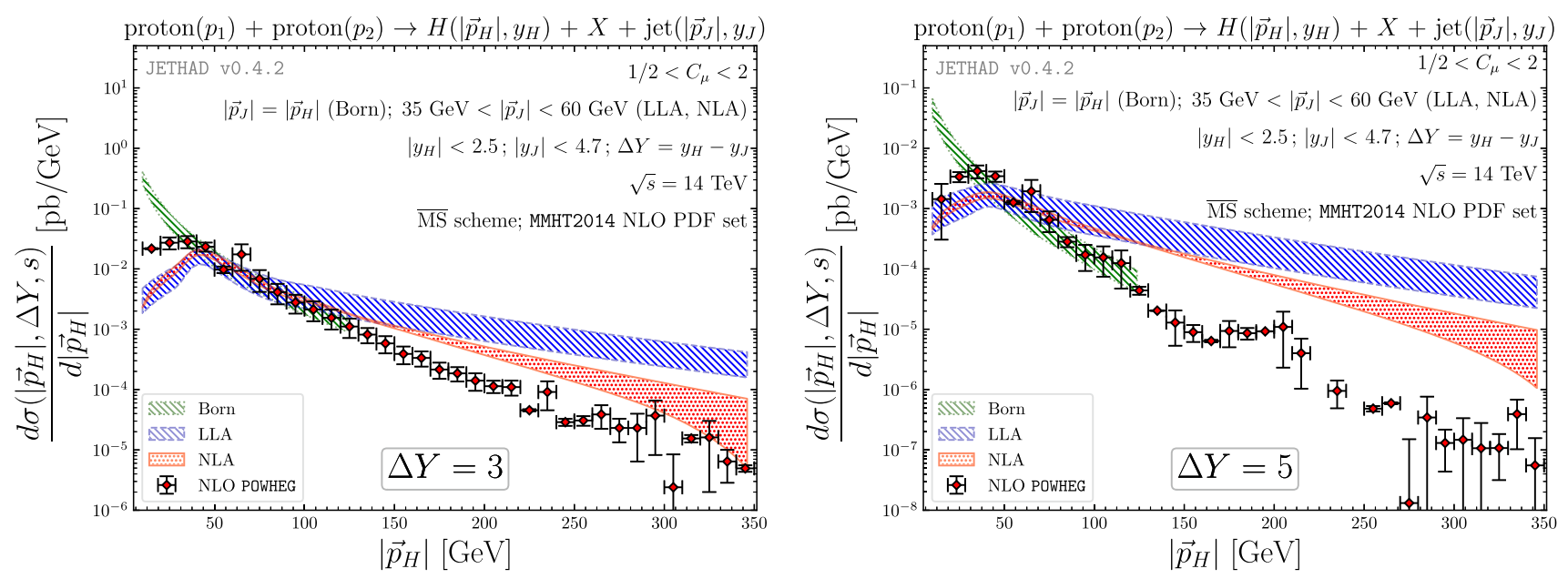

Fig. $8 p_{T}$-dependence of the cross section for the inclusive Higgs-jet hadroproduction for $35 \mathrm{GeV}<\left|\vec{p}_{J}\right|<60 \mathrm{GeV}, \sqrt{s}=14 \mathrm{TeV}$ and for $\Delta Y=3,5$. Shaded bands give the combined effect of the scale variation with the uncertainty coming from the phase-space numerical integration

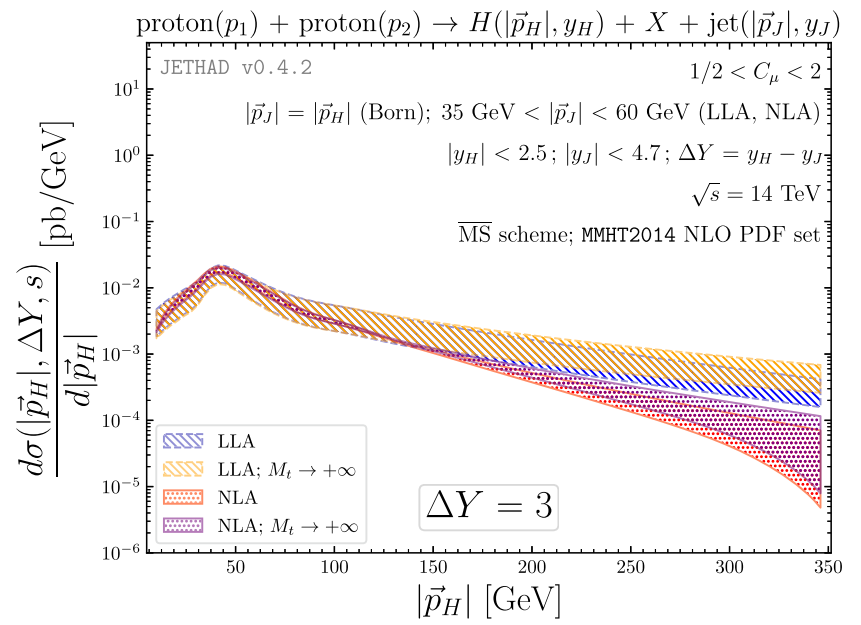

Fig. $9 p_{T}$-dependence of the cross section for the inclusive Higgs-jet hadroproduction in the standard case and in the large top-mass limit, for $35 \mathrm{GeV}<\left|\vec{p}_{J}\right|<60 \mathrm{GeV}, \sqrt{s}=14 \mathrm{TeV}$ and for $\Delta Y=3,5$.

encouraging. At variance with previous analyses, where other kinds of final states were investigated, cross sections and azimuthal correlations for the Higgs-jet production exhibit quite a fair stability under higher-order corrections. Future analyses are needed in order to gauge the feasibility of precision calculations of the same observables. We have extended our study to distributions differential in the Higgs transverse momentum, providing evidence that a high-energy treatment is valid and can be afforded in the region where Higgs $p_{T}$ and the jet one are of the same order.

An obvious extension of this work consists in the full NLA BFKL analysis, including a NLO jet impact factor, with a realistic implementation of the jet selection function, and the NLO Higgs impact factor, when available.

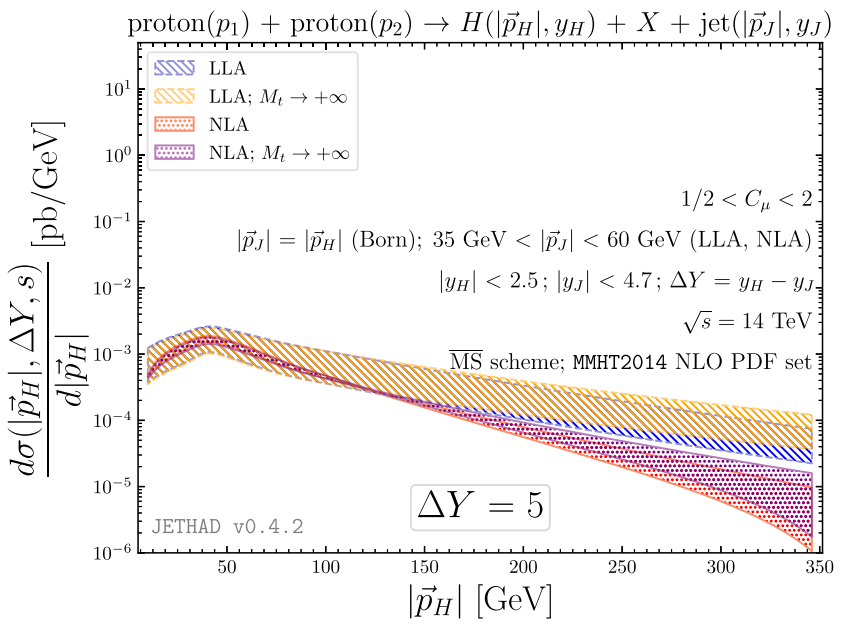

Shaded bands give the combined effect of the scale variation with the uncertainty coming from the phase-space numerical integration

Acknowledgements We thank V. Bertone, G. Bozzi and L. Motyka for helpful discussions and F. Piccinini, C. Del Pio for help on the use of the POWHEG code. FGC acknowledges support from the Italian Ministry of Education, Universities and Research under the FARE grant "3DGLUE" (n. R16XKPHL3N) and from the INFN/NINPHA project. MMAM and AP acknowledge support from the INFN/QFT@colliders project. The work of DI was carried out within the framework of the state contract of the Sobolev Institute of Mathematics (Project No. 03142019-0021).

Data Availability Statement This manuscript has no associated data or the data will not be deposited. [Authors' comment: Data produced in this work are summarized in figures; they can be made available upon request.]

Open Access This article is licensed under a Creative Commons Attribution 4.0 International License, which permits use, sharing, adaptation, distribution and reproduction in any medium or format, as long as you give appropriate credit to the original author(s) and the source, pro- 
vide a link to the Creative Commons licence, and indicate if changes were made. The images or other third party material in this article are included in the article's Creative Commons licence, unless indicated otherwise in a credit line to the material. If material is not included in the article's Creative Commons licence and your intended use is not permitted by statutory regulation or exceeds the permitted use, you will need to obtain permission directly from the copyright holder. To view a copy of this licence, visit http://creativecomm ons.org/licenses/by/4.0/.

Funded by SCOAP ${ }^{3}$.

\section{References}

1. V.S. Fadin, E. Kuraev, L. Lipatov, Phys. Lett. B 60, 50 (1975)

2. V.S. Fadin, E. Kuraev, L. Lipatov, Sov. Phys. JETP 44, 443 (1976)

3. E. Kuraev, L. Lipatov, V.S. Fadin, Sov. Phys. JETP 45, 199 (1977)

4. I. Balitsky, L. Lipatov, Sov. J. Nucl. Phys. 28, 822 (1978)

5. L.V. Gribov, E.M. Levin, M.G. Ryskin, Phys. Rep. 100, 1 (1983)

6. V.S. Fadin, AIP Conf. Proc. 1819(1), 060003 (2017). arXiv: 1612.04481 [hep-ph]

7. V.S. Fadin, L.N. Lipatov, Phys. Lett. B 429, 127 (1998). arXiv: hep-ph/9802290

8. M. Ciafaloni, G. Camici, Phys. Lett. B 430, 349 (1998). arXiv:hep-ph/9803389

9. V.S. Fadin, R. Fiore, A. Papa, Phys. Rev. D 60, 074025 (1999). arXiv:hep-ph/9812456

10. V.S. Fadin, D.A. Gorbachev, Pisma v Zh. Eksp. Teor. Fiz. 71, 322 (2000) [JETP Lett. 71, 222 (2000)]

11. V.S. Fadin, D.A. Gorbachev, Phys. Atom. Nucl. 63, 2157 (2000) [Yad. Fiz. 63, 2253 (2000)]

12. V.S. Fadin, R. Fiore, Phys. Lett. B 610 (2005) 61. arXiv:hep-ph/0412386 [Erratum-ibid. 621, 61 (2005)]

13. V.S. Fadin, R. Fiore, Phys. Rev. D 72, 014018 (2005) arXiv:hep-ph/0502045

14. V.S. Fadin, R. Fiore, M.I. Kotsky, A. Papa, Phys. Rev. D 61, 094005 (2000). arXiv:hep-ph/9908264

15. V.S. Fadin, R. Fiore, M.I. Kotsky, A. Papa, Phys. Rev. D 61, 094006 (2000). arXiv:hep-ph/9908265

16. M. Ciafaloni, D. Colferai, Nucl. Phys. B 538, 187 (1999). arXiv:hep-ph/9806350

17. M. Ciafaloni, G. Rodrigo, JHEP 0005, 042 (2000). arXiv:hep-ph/0004033

18. J. Bartels, D. Colferai, G.P. Vacca, Eur. Phys. J. C 24, 83 (2002). arXiv:hep-ph/0112283

19. J. Bartels, D. Colferai, G.P. Vacca, Eur. Phys. J. C 29, 235 (2003). arXiv:hep-ph/0206290

20. F. Caporale, D.Yu. Ivanov, B. Murdaca, A. Papa, A. Perri, JHEP 1202, 101 (2012). arXiv:1112.3752 [hep-ph]

21. D.Yu. Ivanov, A. Papa, JHEP 1205, 086 (2012). arXiv:1202.1082 [hep-ph]

22. D. Colferai, A. Niccoli, JHEP 1504, 071 (2015). arXiv:1501.07442. [hep-ph]

23. D.Yu. Ivanov, A. Papa, JHEP 1207, 045 (2012). arXiv:1205.6068 [hep-ph]

24. D.Yu. Ivanov, M.I. Kotsky, A. Papa, Eur. Phys. J. C 38, 195 (2004). arXiv:hep-ph/0405297

25. J. Bartels, S. Gieseke, C.F. Qiao, Phys. Rev. D 63, 056014 (2001). arXiv:hep-ph/0009102 [Erratum-ibid. 65, 079902 (2002)]

26. J. Bartels, S. Gieseke, A. Kyrieleis, Phys. Rev. D 65, 014006 (2002). arXiv:hep-ph/0107152

27. J. Bartels, D. Colferai, S. Gieseke, A. Kyrieleis, Phys. Rev. D 66, 094017 (2002). arXiv:hep-ph/0208130

28. J. Bartels, Nucl. Phys. (Proc. Suppl.) 116 (2003)
29. J. Bartels, A. Kyrieleis, Phys. Rev. D 70, 114003 (2004). arXiv:hep-ph/0407051

30. V.S. Fadin, D.Yu. Ivanov, M.I. Kotsky, Phys. Atom. Nucl. 65, 1513 (2002). arXiv:hep-ph/0106099 [Yad. Fiz. 65, 1551 (2002)]

31. V.S. Fadin, D.Yu. Ivanov, M.I. Kotsky, Nucl. Phys. B 658, 156 (2003). arXiv:hep-ph/0210406

32. I. Balitsky, G.A. Chirilli, Phys. Rev. D 87, 014013 (2013). arXiv:hep-ph/1207.3844 [hep-ph]

33. F.G. Celiberto, Ph.D. thesis. arXiv:1707.04315 [hep-ph]

34. F.G. Celiberto, arXiv:2008.07378 [hep-ph]

35. D.Yu. Ivanov, M.I. Kotsky, A. Papa, Eur. Phys. J. C 38, 195 (2004). arXiv:hep-ph/0405297

36. D.Yu. Ivanov, A. Papa, Nucl. Phys. B 732, 183 (2006). arXiv:hep-ph/0508162

37. D.Yu. Ivanov, A. Papa, Eur. Phys. J. C 49, 947 (2007). arXiv:hep-ph/0610042

38. R. Enberg, B. Pire, L. Szymanowski, S. Wallon, Eur. Phys. J. C 45, 759 (2006) [Erratum: Eur. Phys. J. C 51, 1015 (2007)]. arXiv:hep-ph/0508134

39. D.Yu. Ivanov, B. Murdaca, A. Papa, JHEP 10, 058 (2014). arXiv: 1407.8447 [hep-ph]

40. A.H. Mueller, H. Navelet, Nucl. Phys. B 282, 727 (1987)

41. C. Marquet, C. Royon, Phys. Rev. D 79, 034028 (2009). arXiv:0704.3409 [hep-ph]

42. D. Colferai, F. Schwennsen, L. Szymanowski, S. Wallon, JHEP 1012, 026 (2010). arXiv:1002.1365 [hep-ph]

43. F. Caporale, D.Yu. Ivanov, B. Murdaca, A. Papa, Nucl. Phys. B 877, 73 (2013). arXiv:1211.7225 [hep-ph]

44. B. Ducloué, L. Szymanowski, S. Wallon, JHEP 1305, 096 (2013). arXiv:1302.7012 [hep-ph]

45. B. Ducloué, L. Szymanowski, S. Wallon, Phys. Rev. Lett. 112, 082003 (2014). arXiv:1309.3229 [hep-ph]

46. F. Caporale, B. Murdaca, A. Sabio Vera, C. Salas, Nucl. Phys. B 875, 134 (2013). arXiv:1305.4620 [hep-ph]

47. B. Ducloué, L. Szymanowski, S. Wallon, Phys. Lett. B 738, 311 (2014). arXiv:1407.6593 [hep-ph]

48. F. Caporale, D.Yu. Ivanov, B. Murdaca, A. Papa, Eur. Phys. J. C 74(10), 3084 (2014) [Eur. Phys. J. C 75(11), 535 (2015)]. arXiv:1407.8431 [hep-ph]

49. B. Ducloué, L. Szymanowski, S. Wallon, Phys. Rev. D 92(7), 076002 (2015). arXiv:1507.04735 [hep-ph]

50. F. Caporale, D.Yu. Ivanov, B. Murdaca, A. Papa, Phys. Rev. D 91(11), 114009 (2015). arXiv:1504.06471 [hep-ph]

51. F.G. Celiberto, D.Yu. Ivanov, B. Murdaca, A. Papa, Eur. Phys. J. C 75(6), 292 (2015). arXiv:1504.08233 [hep-ph]

52. F.G. Celiberto, D.Yu. Ivanov, B. Murdaca, A. Papa, Acta Phys. Polon. Suppl. 8, 935 (2015). arXiv:1510.01626 [hep-ph]

53. F.G. Celiberto, D.Yu. Ivanov, B. Murdaca, A. Papa, Eur. Phys. J. C 76(4), 224 (2016). arXiv:1601.07847 [hep-ph]

54. F.G. Celiberto, D. Yu. Ivanov, B. Murdaca, A. Papa, PoS DIS 2016 , 176 (2016). arXiv:1606.08892 [hep-ph]

55. F. Caporale, F.G. Celiberto, G. Chachamis, D. Gordo Gómez, A. Sabio Vera, Nucl. Phys. B 935, 412 (2018). arXiv:1806.06309 [hep-ph]

56. G. Chachamis, arXiv:1512.04430 [hep-ph]

57. D. Colferai, A. Niccoli, F. Deganutti, PoS QCDEV2016, 031 (2017)

58. F.G. Celiberto, D.Yu. Ivanov, B. Murdaca, A. Papa, Phys. Rev. D 94(3), 034013 (2016). arXiv:1604.08013 [hep-ph]

59. F.G. Celiberto, D.Yu. Ivanov, B. Murdaca, A. Papa, AIP Conf. Proc. 1819(1), 060005 (2017). arXiv:1611.04811 [hep-ph]

60. F.G. Celiberto, D.Yu. Ivanov, B. Murdaca, A. Papa, Eur. Phys. J. C 77(6), 382 (2017). arXiv:1701.05077 [hep-ph]

61. F. Caporale, G. Chachamis, B. Murdaca, A. Sabio Vera, Phys. Rev. Lett. 116(1), 012001 (2016). arXiv:1508.07711 [hep-ph] 
62. F. Caporale, F.G. Celiberto, G. Chachamis, A. Sabio Vera, Eur. Phys. J. C 76(3), 165 (2016). arXiv:1512.03364 [hep-ph]

63. F. Caporale, F.G. Celiberto, G. Chachamis, D.Gordo Gómez, A. Sabio Vera, Nucl. Phys. B 910, 374 (2016). arXiv:1603.07785 [hep-ph]

64. F. Caporale, F.G. Celiberto, G. Chachamis, A. Sabio Vera, PoS DIS 2016, 177 (2016). arXiv:1610.01880 [hep-ph]

65. F. Caporale, F.G. Celiberto, G. Chachamis, D.Gordo Gómez, A. Sabio Vera, Eur. Phys. J. C 77(1), 5 (2017). arXiv:1606.00574 [hep-ph]

66. F.G. Celiberto, Frascati Phys. Ser. 63, 43 (2016). arXiv:1606.07327 [hep-ph]

67. F. Caporale, F.G. Celiberto, G. Chachamis, D. Gordo Gomez, A. Sabio Vera, AIP Conf. Proc. 1819(1), 060009 (2017). arXiv:1611.04813 [hep-ph]

68. F. Caporale, F.G. Celiberto, G. Chachamis, D. Gordo Gómez, A. Sabio Vera, EPJ Web Conf. 164, 07027 (2017). arXiv:1612.02771 [hep-ph]

69. F. Caporale, F.G. Celiberto, G. Chachamis, D. Gordo Gómez, A. Sabio Vera, Phys. Rev. D 95(7), 074007 (2017). arXiv:1612.05428 [hep-ph]

70. R. Boussarie, B. Ducloué, L. Szymanowski, S. Wallon, Phys. Rev. D 97(1), 014008 (2018). arXiv:1709.01380 [hep-ph]

71. A.D. Bolognino, F.G. Celiberto, D.Yu. Ivanov, M.M.A. Mohammed, A. Papa, Eur. Phys. J. C 78(9), 772 (2018). arXiv: 1808.05483 [hep-ph]

72. A.D. Bolognino, F.G. Celiberto, D.Yu. Ivanov, M.M.A. Mohammed, A. Papa, Acta Phys. Polon. Suppl. 12(4), 773 (2019). arXiv:1902.04511 [hep-ph]

73. A.D. Bolognino, F.G. Celiberto, D.Yu. Ivanov, M.M.A. Mohammed, A. Papa, PoS DIS 2019, 049 (2019). arXiv:1906.11800 [hep-ph]

74. F.G. Celiberto, D.Yu. Ivanov, A. Papa, Phys. Rev. D 102(9), 094019 (2020). arXiv:2008.10513 [hep-ph]

75. K. Golec-Biernat, L. Motyka, T. Stebel, JHEP 1812, 091 (2018). arXiv:1811.04361 [hep-ph]

76. M. Deak, A. van Hameren, H. Jung, A. Kusina, K. Kutak, M. Serino, Phys. Rev. D 99(9), 094011 (2019). arXiv:1809.03854 [hep-ph]

77. F.G. Celiberto, D.Yu. Ivanov, B. Murdaca, A. Papa, Phys. Lett. B 777, 141 (2018). arXiv:1709.10032 [hep-ph]

78. A.D. Bolognino, F.G. Celiberto, M. Fucilla, D. Yu. Ivanov, B. Murdaca, A. Papa, PoS DIS 2019, 067 (2019). arXiv:1906.05940 [hep$\mathrm{ph}]$

79. A.D. Bolognino, F.G. Celiberto, M. Fucilla, D.Yu. Ivanov, A. Papa, Eur. Phys. J. C 79(11), 939 (2019). arXiv:1909.03068 [hep$\mathrm{ph}]$

80. M. Hentschinski, A. Sabio Vera, C. Salas, Phys. Rev. Lett. 110(4), 041601 (2013). arXiv:1209.1353 [hep-ph]

81. A. Besse, L. Szymanowski, S. Wallon, JHEP 1311, 062 (2013). arXiv:1302.1766 [hep-ph]

82. A.D. Bolognino, F.G. Celiberto, D.Yu. Ivanov, A. Papa, Eur. Phys. J. C 78(12), 1023 (2018). arXiv:1808.02395 [hep-ph]

83. A.D. Bolognino, F.G. Celiberto, D.Yu. Ivanov, A. Papa, Frascati Phys. Ser. 67, 76 (2018). arXiv:1808.02958 [hep-ph]

84. A.D. Bolognino, F.G. Celiberto, D.Yu. Ivanov, A. Papa, Acta Phys. Polon. Suppl. 12(4), 891 (2019). arXiv:1902.04520 [hep$\mathrm{ph}]$

85. F.G. Celiberto, Nuovo Cim. C 42, 220 (2019). arXiv:1912.11313 [hep-ph]

86. A.D. Bolognino, A. Szczurek, W. Schäfer, Phys. Rev. D 101(5), 054041 (2020). arXiv:1912.06507 [hep-ph]

87. L. Motyka, M. Sadzikowski, T. Stebel, JHEP 1505, 087 (2015). arXiv: 1412.4675 [hep-ph]

88. D. Brzeminski, L. Motyka, M. Sadzikowski, T. Stebel, JHEP 1701, 005 (2017). arXiv:1611.04449 [hep-ph]
89. F.G. Celiberto, D. Gordo Gómez, A. Sabio Vera, Phys. Lett. B 786, 201 (2018). arXiv:1808.09511 [hep-ph]

90. R.D. Ball, V. Bertone, M. Bonvini, S. Marzani, J. Rojo, L. Rottoli, Eur. Phys. J. C 78(4), 321 (2018). arXiv:1710.05935 [hep-ph]

91. H. Abdolmaleki et al. [xFitter Developers' Team], Eur. Phys. J. C 78(8), 621 (2018). arXiv:1802.00064 [hep-ph]

92. M. Bonvini, F. Giuli, Eur. Phys. J. Plus 134(10), 531 (2019). arXiv:1902.11125 [hep-ph]

93. A. Bacchetta, F.G. Celiberto, M. Radici, P. Taels, Eur. Phys. J. C 80(8), 733 (2020). arXiv:2005.02288 [hep-ph]

94. B.W. Xiao, F. Yuan, Phys. Lett. B 782, 28 (2018). arXiv:1801.05478 [hep-ph]

95. V. Del Duca, C.R. Schmidt, Phys. Rev. D 49, 177 (1994) arXiv:hep-ph/9305346

96. A.H. Mueller, L. Szymanowski, S. Wallon, B.W. Xiao, F. Yuan, JHEP 03, 096 (2016). arXiv:1512.07127 [hep-ph]

97. P.F. Monni, E. Re, P. Torrielli, Phys. Rev. Lett. 116(24), 242001 (2016). arXiv:1604.02191 [hep-ph]

98. W. Bizoń, P.F. Monni, E. Re, L. Rottoli, P. Torrielli, JHEP 02, 108 (2018). arXiv:1705.09127 [hep-ph]

99. W. Bizoń, X. Chen, A. Gehrmann-De Ridder, T. Gehrmann, N. Glover, A. Huss, P.F. Monni, E. Re, L. Rottoli, P. Torrielli, JHEP 12, 132 (2018). arXiv:1805.05916 [hep-ph]

100. X. Chen, T. Gehrmann, E.W.N. Glover, A. Huss, Y. Li, D. Neill, M. Schulze, I.W. Stewart, H.X. Zhu, Phys. Lett. B 788, 425-430 (2019). arXiv:1805.00736 [hep-ph]

101. P.F. Monni, L. Rottoli, P. Torrielli, Phys. Rev. Lett. 124(25), 252001 (2020). arXiv:1909.04704 [hep-ph]

102. M. Hentschinski, K. Kutak, A. van Hameren, Eur. Phys. J. C 81(2), 112 (2021). arXiv:2011.03193 [hep-ph]

103. M. Bonvini, S. Marzani, Phys. Rev. Lett. 120(20), 202003 (2018). arXiv:1802.07758 [hep-ph]

104. M. Bonvini, Eur. Phys. J. C 78(10), 834 (2018). arXiv: 1805.08785 [hep-ph]

105. R.D. Ball, S. Forte, Phys. Lett. B 351, 313 (1995). arXiv:hep-ph/9501231

106. R.D. Ball, S. Forte, Phys. Lett. B 405, 317 (1997). arXiv:hep-ph/9703417

107. G. Altarelli, R.D. Ball, S. Forte, Nucl. Phys. B 621, 359 (2002). arXiv:hep-ph/0109178

108. G. Altarelli, R.D. Ball, S. Forte, Nucl. Phys. B 674, 459 (2003) arXiv:hep-ph/0306156

109. G. Altarelli, R.D. Ball, S. Forte, Nucl. Phys. B 742, 1 (2006). arXiv:hep-ph/0512237

110. G. Altarelli, R.D. Ball, S. Forte, Nucl. Phys. B 799, 199 (2008). arXiv:0802.0032 [hep-ph]

111. C. White, R. Thorne, Phys. Rev. D 75, 034005 (2007). arXiv:hep-ph/0611204 [hep-ph]

112. F. Hautmann, Phys. Lett. B 535, 159-162 (2002). arXiv:hep-ph/0203140 [hep-ph]

113. A. Sabio Vera, Nucl. Phys. B 746, 1-14 (2006) arXiv:hep-ph/0602250 [hep-ph]

114. A. Sabio Vera, F. Schwennsen, Nucl. Phys. B 776, 170-186 (2007). arXiv:hep-ph/0702158 [hep-ph]

115. R. Bonciani, V. Del Duca, H. Frellesvig, J.M. Henn, F. Moriello, V.A. Smirnov, JHEP 1612, 096 (2016). arXiv:1609.06685 [hep$\mathrm{ph}]$

116. S.P. Jones, M. Kerner, G. Luisoni, Phys. Rev. Lett. 120(16), 162001 (2018). arXiv:1802.00349 [hep-ph]

117. R. Boughezal, F. Caola, K. Melnikov, F. Petriello, M. Schulze, JHEP 1306, 072 (2013). arXiv:1302.6216 [hep-ph]

118. X. Chen, T. Gehrmann, E.W.N. Glover, M. Jaquier, Phys. Lett. B 740, 147 (2015). arXiv:1408.5325 [hep-ph]

119. R. Boughezal, F. Caola, K. Melnikov, F. Petriello, M. Schulze, Phys. Rev. Lett. 115(8), 082003 (2015). arXiv:1504.07922 [hep$\mathrm{ph}]$ 
120. R. Boughezal, C. Focke, W. Giele, X. Liu, F. Petriello, Phys. Lett. B 748, 5 (2015). arXiv: 1505.03893 [hep-ph]

121. F. Wilczek, Phys. Rev. Lett. 39, 1304 (1977)

122. V.S. Fadin, R. Fiore, Phys. Lett. B 440, 359-366 (1998). arXiv:hep-ph/9807472 [hep-ph]

123. V. Del Duca, W. Kilgore, C. Oleari, C.R. Schmidt, D. Zeppenfeld, Phys. Rev. D 67, 073003 (2003). arXiv:hep-ph/0301013

124. M. Tanabashi et al. [Particle Data Group], Phys. Rev. D 98(3), 030001 (2018)

125. V. Khachatryan et al. [CMS Collaboration], JHEP 1608, 139 (2016). arXiv:1601.06713 [hep-ex]

126. P. Cipriano, S. Dooling, A. Grebenyuk, P. Gunnellini, F. Hautmann, H. Jung, P. Katsas, Phys. Rev. D 88(9), 097501 (2013). arXiv:1308.1655 [hep-ph]

127. Y.L. Dokshitzer, D. Diakonov, S.I. Troian, Phys. Lett. B 79, 269 (1978)

128. Y.L. Dokshitzer, D. Diakonov, S.I. Troian, Phys. Rep. 58, 269 (1980)

129. G. Parisi, R. Petronzio, Nucl. Phys. B 154, 427 (1979)

130. G. Curci, M. Greco, Y. Srivastava, Nucl. Phys. B 159, 451 (1979)

131. J.C. Collins, D.E. Soper, Nucl. Phys. B 193, 381 (1981) [Erratum: Nucl. Phys. B 213, 545 (1983)]

132. J.C. Collins, D.E. Soper, Nucl. Phys. B 197, 446 (1982)

133. J.C. Collins, D.E. Soper, G.F. Sterman, Nucl. Phys. B 250, 199 (1985)

134. J. Kodaira, L. Trentadue, Phys. Lett. B 112, 66 (1982)

135. J. Kodaira, L. Trentadue, Nucl. Phys. B (submitted)

136. J. Kodaira, L. Trentadue, Phys. Lett. B 123, 335 (1983)

137. S. Catani, D. de Florian, M. Grazzini, Nucl. Phys. B 596, 299 (2001). arXiv:hep-ph/0008184

138. G. Bozzi, S. Catani, D. de Florian, M. Grazzini, Nucl. Phys. B 737, 73 (2006). arXiv:hep-ph/0508068
139. G. Bozzi, S. Catani, G. Ferrera, D. de Florian, M. Grazzini, Nucl. Phys. B 815, 174 (2009). arXiv:0812.2862 [hep-ph]

140. S. Catani, M. Grazzini, Nucl. Phys. B 845, 297 (2011). arXiv:1011.3918 [hep-ph]

141. S. Catani, M. Grazzini, Eur. Phys. J. C 72, 2013 (2012) [Erratum: Eur. Phys. J. C 72, 2132 (2012)]. arXiv:1106.4652 [hep-ph]

142. S. Catani, L. Cieri, D. de Florian, G. Ferrera, M. Grazzini, Nucl. Phys. B 881, 414 (2014). arXiv:1311.1654 [hep-ph]

143. S. Catani, D. de Florian, G. Ferrera, M. Grazzini, JHEP 1512, 047 (2015). arXiv:1507.06937 [hep-ph]

144. R. Bonciani, S. Catani, M.L. Mangano, P. Nason, Phys. Lett. B 575, 268 (2003). arXiv:hep-ph/0307035

145. D. de Florian, A. Kulesza, W. Vogelsang, JHEP 0602, 047 (2006). arXiv:hep-ph/0511205

146. C. Muselli, S. Forte, G. Ridolfi, JHEP 1703, 106 (2017). arXiv: 1701.01464 [hep-ph]

147. P. Nason, JHEP 11, 040 (2004). arXiv:hep-ph/0409146

148. S. Frixione, P. Nason, C. Oleari, JHEP 11, 070 (2007). arXiv:0709.2092 [hep-ph]

149. S. Frixione, P. Nason, C. Oleari, JHEP 06, 043 (2010). arXiv:1002.2581 [hep-ph]

150. J.M. Campbell, R.K. Ellis, R. Frederix, P. Nason, C. Oleari, C. Williams, JHEP 07, 092 (2012). arXiv: 1202.5475 [hep-ph]

151. K. Hamilton, P. Nason, C. Oleari, G. Zanderighi, JHEP 05, 082 (2013). arXiv:1212.4504 [hep-ph]

152. L.A. Harland-Lang, A.D. Martin, P. Motylinski, R.S. Thorne, Eur. Phys. J. C 75(5), 204 (2015). arXiv:1412.3989 [hep-ph]

153. A. Buckley, J. Ferrando, S. Lloyd, K. Nordström, B. Page, M. Rüfenacht, M. Schönherr, G. Watt, Eur. Phys. J. C 75, 132 (2015). arXiv:1412.7420 [hep-ph] 